\title{
Using Formal Concept Analysis Tools in Road Environment-Type Detection
}

\author{
A. Boulmakoul ${ }^{1(\otimes)}$, Z. Fazekas ${ }^{2}$, L. Karim ${ }^{1,3}$, G. Cherradi ${ }^{1}$, and P. Gáspár ${ }^{2}$ \\ 1 Computer Science Department, FSTM, Hassan II University of Casablanca, \\ Casablanca, Morocco \\ azedine.boulmakoul@gmail.com, cherradighyzlane@gmail.com \\ 2 Institute for Computer Science and Control (SZTAKI), Budapest, Hungary \\ fazekas.zoltan@sztaki.mta.hu \\ 3 LISA Laboratory, ENSA Berrechid, Hassan 1st University, Settat, Morocco \\ lkarim.lkarim@gmail.com
}

\begin{abstract}
This paper presents an urban road environment-type (RET) detection algorithm for an ego-vehicle that is equipped with an automatic traffic sign recognition (TSR) system. The RETs considered in the paper are the downtown, the residential, and the business/industrial areas. The Galois-lattice (GL) often used in formal concept analysis (FCA) is employed to specify the RET detection problem in a formal manner. With the help of the GL, a correspondence between the traffic sign (TS) and crossroad (CR) categories, on the one hand, and the RETs, on the other, is established and represented. This correspondence is also characterized by means of a fuzzy matrix structure, and its alpha-cuts. After an FCA-based learning process, in the detection phase, i.e., while the ego-car is driven along the route, the maximum couplings between RETs and TS/CR sets are searched for in an on-the-fly manner. The proposed RET detection method is suitable for real-time implementation. The paper underlines the importance and applicability of GLs in classification problems.
\end{abstract}

Keywords: Road environment-type detection - Fuzzy sets • Galois-lattice $\cdot$ Formal concept analysis $\cdot$ Knowledge discovery and representation

\section{Introduction}

A smart vehicle is capable of detecting its environment, and navigate along a route either with the help of the driver, i.e., being driven by the driver, or indeed all by its own, i.e., in total autonomy. The available technologies rely on various input data streams. These streams can be generated by one or more camera, one or more infrared sensor, one or more LiDAR, one or more radar, etc. Current research developments produce innovative sensor fusion solutions 
that build upon computer vision, sensor networks, machine learning and artificial intelligence techniques, including deep learning approaches, see e.g., [12].

A traffic sign (TS) based urban road environment-type (RET) detection system was proposed in [5]. The RET detection system gathers TS data along the route with the help of the TSR system, and employs a stochastic change detection method in respect of the collected TS data to identify RET change-points, i.e., certain road locations, along a route. Various variants of TS-based RET detection system was proposed later, e.g., [6-8] and [9]. These detection methods rely on different computing techniques ranging from the shallow artificial neural networks (ANNs) to the heuristic rule-based decision making techniques. Some of these approaches use additional input data types, such as types and location of the encountered crossroads (CRs) along the route. Clearly, numerous other input data-types could be employed for the purpose, e.g., number of lanes along the route, width of the actual road/lane.

Galois lattices (GL) are algebraic structures related to the order theory, see e.g., $[3,4]$. Galois structures and concept lattices are key structures of the formal concept analysis (FCA), [10,13], which is a widely used data mining methodology for formal contexts composed of objects, which are in turn described by attributes. A good introduction to GLs is given by [1] via a fairly common educational example: evaluation of class-test results by a teacher. The educational and test-design conclusions are also interesting for an educational practitioner, including those working in the higher education.

Closer to our present topic of detecting the road environment, in particular, the road environment meant in a socio-economic sense and viewed at a corresponding scale, a knowledge-based agricultural landscape analysis framework was proposed in [11]. The GL-based framework presented there includes a concept lattice structure of topological relations for qualitative spatial reasoning. The main objective was to recognize landscape models on land-use maps extracted from satellite images. These models are abstract models describing agricultural spatial structures as sets of spatial entities and qualitative spatial relations between these entities. The map-regions, or zones, can represent certain agricultural environments, such as crops, meadows, forest, buildings, etc.

In the present paper, a GL is employed to capture the correspondence between traffic signs (TSs) and crossroads categories (CRs), on the one hand, and urban RETs, on the other. The three urban RETs considered are the downtown, residential, and business/industrial areas. These characterize the road environments around the ego-car, and provide a hint for the driver about the traffic safety risks associated with such areas. The detection approach presented here takes into account only certain - i.e., relevant for the purpose - TS types. Furthermore, for a TS to be considered in the computations, it needs to be visible/detectable from the ego-car moving along its route.

As a first processing step, fuzzy sets are created based on the occurrence probabilities of the TS types within the urban RETs. Then, the correspondence between TSs and urban RETs - by means of the alpha-cuts applied to the fuzzy matrix built in the previous step - are established. Then, a learning process based on a FCA method - explores and highlights the maximum couplings that 
exsist between a TSs and the RETs. In the next processing step, these maximum couplings are used in the inference of the actual RET. A new, efficient closed sets exploration procedure for characterizing the individual RETs is proposed in the paper to support the inference.

The remainder of the paper is structured as follows. Section 2 provides a brief recall of the fuzzy concepts used. In Sect. 3, an FCA-based RET detection approach is described, while in Sect. 4 , the software architecture of the implemented prototype, as well as the methodology of its deployment is covered. A simple, but illustrative example is given to show the main processing steps. Finally, in Sect. 5 , the conclusions are drawn.

\section{Preliminaries}

This work focuses on the algebraic calculations - carried out within and with the help of GL structures - to reason about the relationships between the elements of a domain, e.g., various TSs, CRs and the considered RETs. It also makes use of the fuzzy sets and related concepts. The notations and the necessary definitions for understanding the rest of the paper are given in this section. We recall the definitions of fuzzy set and GL concepts based on $[2,14,15]$.

\subsection{Fuzzy Concepts}

Definition 1: If $\mathrm{X}$ is a collection of objects denoted generically by $x$, then a fuzzy set $\widetilde{A}$ in $\mathrm{X}$ is a set of ordered pairs.

$$
\widetilde{A}=\left\{x, \mu_{\widetilde{A}}(x) \mid x \in X\right\}
$$

$\mu_{\widetilde{A}}(x)$ is the membership function, or grade - in some publications also referred to as the degree of compatibility, or the degree of truth - of $x$ in $\widetilde{A}$ that maps $X$ to the membership space $M$ (When $M=\{0,1\}, \widetilde{A}$ is crisp set and $\mu_{\widetilde{A}}(x)$ is identical to the characteristic function of non-fuzzy set).

The (crisp) set of elements that belong to the fuzzy set $\widetilde{A}$ at least to the degree $\alpha$ is called the $\alpha$-level set:

$$
A_{\alpha}=\left\{x \in X \mid \mu_{\widetilde{A}}(x) \geq \alpha\right\}
$$

$A_{\alpha}^{\prime}=\left\{x \in X \mid \mu_{\widetilde{A}}(x)>\alpha\right\}$ is called "strong $\alpha$ - level set" or "strong $\alpha-$ cut". Throughout this paper, the following notations have been used. $\mathbb{R}^{+}=$ $[0, \infty) ; X=\left\{x_{1}, x_{2}, \ldots, x_{n}\right\}$ is the universal set; $\mathcal{F}(X)$ is the class of all fuzzy sets of $\mathrm{X} ; \mu_{A}\left(x_{i}\right): X \longrightarrow[0.1]$ is the membership function of $A \in \mathcal{F}(X) ; A^{c} \in \mathcal{F}$ is the complement of $A \in \mathcal{F}$. 


\subsection{Formal Concept Analysis Concepts}

Definition 2: A formal context $\mathbb{k}:=(G, M, I)$ consists of two sets $G$ and $M$ and a relation $I$ between $G$ and $M$. The elements of $G$ are called the objects and the elements of $M$ are called the attributes of the context. In order to express that an object $\mathrm{g}$ is in a relation $I$ with an attribute $\mathrm{m}$,we write $\mathrm{gIm}$ or $(g, m) \in I$ and read it as "the object $g$ has the attribute $m$ " [4,10,13].

Definition 3: For a set $A \subseteq G$ of objects we define

$$
A^{\prime}=\{m \in M \mid g I m \text { for all } g \in A\}
$$

(the set of attributes common to the objects in $A$ ). Correspondingly, for a set $B$ of attributes we define

$$
B^{\prime}=\{g \in G \mid g I m \text { for all } m \in B\}
$$

(the set of objects which have all attributes in $B$ ).

Definition 4: a formal concept of the context $(G, M, I)$ is a pair $(A, B)$ with $A \subseteq G, B \subseteq M, A^{\prime}=B$ and $B^{\prime}=A$. We call $A$ the extent and $B$ the intent of the concept $(A, B) . \mathcal{B}(G, M, I)$ denotes the set of all concepts of the context $(G, M, I)$.

Proposition: if $(G, M, I)$ is a context, $A, A_{1}, A_{2} \subseteq G$ are sets of objects and $B, B_{1}, B_{2}$ are sets of attributes. Then :
1) $A_{1} \subseteq A_{2} \Rightarrow A_{2}^{\prime} \subseteq A_{1}^{\prime}$
2) $A \subseteq A^{\prime \prime}$
1) $B_{1} \subseteq B_{2} \Longrightarrow B_{2}^{\prime} \subseteq B_{1}^{\prime}$
3) $A^{\prime}=A^{\prime \prime \prime}$
2) $B \subseteq B^{\prime \prime}$
4) $A \subseteq B^{\prime} \Longleftrightarrow B \subseteq A^{\prime} \Longleftrightarrow A \times B \subseteq I$.

Definition 5: If $\left(A_{1}, B_{1}\right)$ and $\left(A_{2}, B_{2}\right)$ are concepts of a context, $\left(A_{1}, B_{1}\right)$ is called a subconcept of $\left(A_{2}, B_{2}\right)$, provided that $A_{1} \subseteq A_{2}$ (which is equivalent to $\left.B_{2} \subseteq B_{1}\right)$. In this case, $\left(A_{2}, B_{2}\right)$ is a superconcept of $\left(A_{1}, B_{1}\right)$, and we write $\left(A_{1}, B_{1}\right) \leqslant\left(A_{2}, B_{2}\right)$. The relation $\leqslant$ is called the hierarchical order (or simply order) of the concepts. The set of all concepts of $(G, M, I)$ ordered in this way is denoted by $\mathcal{B}(G, M, I)$ and is called the concept lattice of the context $(G, M, I)$.

\subsection{Fuzzy Galois Lattice}

Inaccurate and fuzzy values either measured in physical systems, or detected in, calculated for biological, economic, or social systems inspired great deal of research among others in the field of fuzzy FCA [2], particularly with regard to the generalization of closure operators concerning fuzzy relations and the underlying problem of fuzzy formal concepts generation. 
The FCA consists in inducing formal concepts $<$ Objects, Attributes $>$ from a formal binary context. These formal concepts are based on the following definition: "An object belongs to the Objects subset, if it has all the attributes" [10]. Since the relation considered in the formal context is Boolean, an object has an attribute altogether, or does not have it at all. Indeed, in real problems the FCA is brought to use formal contexts of various nature, obtained by measurements, observations, judgments, etc., where the relation between an object and an attribute can be more uncertain, and less specific. Or even gradual, or imprecise. Various approaches have been proposed for the extension of the FCA to the fuzzy 'worls'.

\section{RET Detection by Means of FCA}

\section{$3.1 \quad$ Notation}

Hereinafter, we describe some useful notations for the rest of the paper.

$E_{i}=\{$ spatial object of layer $(i)\}=\left\{e_{j}^{m, i} \mid j=1 . . n\right\}$ which defines a layer. The set of all layers: $E=\cup_{i} E_{i}$.

$\vartheta_{i}(m, \varepsilon)=\left\{o \in E_{i} \mid \delta(m, o) \leq \varepsilon \bigvee \mathcal{L}(m, o) \vdash\right.$ true $\}$ all the objects of the layer $E_{i}$ which are at a distance $\epsilon$ from the mobile $(\mathrm{i}) . \mathcal{L}(m, o)$ defines a spatial predicate between the mobile $\mathrm{m}$ and the object $\mathrm{o}$.

$$
\mathbb{C}=\text { Road Environment }- \text { Type }(R E T)=\left\{\sigma_{i} \mid i=1 . . m\right\} .
$$

Example:

$\mathbb{C}=\{$ downtown, residential area, business $\mid$ industrial area,$\ldots\}$

$$
\theta_{i, k}^{m, j}=\mathcal{L}\left(\sigma_{i}, e_{k}^{m, j}\right) \vdash t r u e
$$

The context is built by a learning process, e.g., a set of vehicles collects information concerning the RETs and the CR categories along certain routes. Induction type urban area explained by the other variables (according to layer) : $\mathbb{C} \times E_{i}$, ou $\mathbb{C} \times{ }_{j=1}^{j=k} \oplus E_{j}$. This correspondence will be designated by $: \mho_{m}^{\{\ell: \text { layers }\}}$ being the set of topics considered for the context. Induction type urban area explained by the other variables (according to selected layers). We define $\mho_{\triangle}^{\{\ell: \text { layers }\}}={ }_{j=1}^{j=k} \oplus \mho_{m}^{\{\ell: \text { layers }\}}$, where $\oplus$ denotes an algebraic operation on matrices (t-conorm of fuzzy relations sets) $\Lambda^{\alpha}\left(\gamma_{i}, \sigma_{j}\right)=\left\{\begin{array}{l}1 ; \text { if }\left[\wedge\left(\gamma_{i}, \sigma_{j}\right) \geqslant \alpha\right] \\ 0 ; \text { otherwise }\end{array}\right.$

Where:

$$
E_{T S}=\left\{\gamma_{1}, \gamma_{2}, \gamma_{3}, \gamma_{4}\right\} ; E_{C R}=\left\{\gamma_{5}, \gamma_{6}, \gamma_{7}, \gamma_{8}, \gamma_{9}\right\} ; \mathbb{C}=\left\{\sigma_{1}, \sigma_{2}, \sigma_{3}\right\}
$$

Hereafter we illustrate this process by infered fuzzy sets:

$$
\begin{aligned}
& \text { (D) } \sigma_{1}=\left\{\gamma_{1} / 0.22, \gamma_{2} / 0.18, \gamma_{3} / 0.07, \gamma_{4} / 0.02, \gamma_{5} / 0.28, \gamma_{6} / 0.13, \gamma_{7} / 0.01, \gamma_{8} / 0.001, \gamma_{9} / 0.05\right\} \\
& (R) \sigma_{2}=\left\{\gamma_{1} / 0.03, \gamma_{2} / 0.025, \gamma_{3} / 0.08, \gamma_{4} / 0.04, \gamma_{5} / 0.32, \gamma_{6} / 0.08, \gamma_{7} / 0.005, \gamma_{8} / 0.002, \gamma_{9} / 0.02\right\} \\
& \text { (IC) } \sigma_{3}=\left\{\gamma_{1} / 0.04, \gamma_{2} / 0.017, \gamma_{3} / 0.055, \gamma_{4} / 0.02, \gamma_{5} / 0.20, \gamma_{6} / 0.015, \gamma_{7} / 0.001, \gamma_{8} / 0.002, \gamma_{9} / 0.04\right\}
\end{aligned}
$$


Table 1. The probability of occurrence of TS's and CR's - along a $50 \mathrm{~m}$ path-length - in the three urban RE's considered from [6].

\begin{tabular}{|ll|l|l|l|}
\hline & RET & $\begin{array}{l}\sigma_{1} \\
\text { Downtown }\end{array}$ & $\begin{array}{l}\sigma_{2} \\
\text { Residential }\end{array}$ & Industrial/Commercial \\
\hline TS & & & & \\
\hline$\gamma_{1}$ & $\bullet$ & $22 \%$ & $3 \%$ & $4 \%$ \\
\hline$\gamma_{2}$ & $\mathbf{P}$ & $18 \%$ & $2,5 \%$ & $1,7 \%$ \\
\hline$\gamma_{3}$ & $\nabla$ & $7 \%$ & $8 \%$ & $5,5 \%$ \\
\hline$\gamma_{4}$ & $\circledast$ & $2 \%$ & $4 \%$ & $2 \%$ \\
\hline$\gamma_{5}$ & $\mathbf{T}$ & $28 \%$ & $32 \%$ & $20 \%$ \\
\hline$\gamma_{6}$ & + & $13 \%$ & $8 \%$ & $1,5 \%$ \\
\hline$\gamma_{7}$ & $*$ & $1 \%$ & $0,5 \%$ & $0,1 \%$ \\
\hline$\gamma_{8}$ & $-\beta$ & $0,1 \%$ & $0,2 \%$ & $0,2 \%$ \\
\hline$\gamma_{9}$ & $*$ & $5 \%$ & $2 \%$ & $4 \%$ \\
\hline
\end{tabular}

\section{The FCA-based RET Detection Process}

The analysis of formal concepts is an analytical practice based on graph theory and strongly linked to Galois lattices [3]. It is a theory of mathematical algebra that makes it possible to formalize concepts as basic units of human thought and to analyze data in attribute-object form [10]. Created in the early 80's and over the last three decades, it has become a popular engineering tool for humancentered knowledge representation and data analysis with many applications in many fields. In this section, we rely on the GL foundations, [3,4], and illustrate with a simple example the use and the interpretation of its elements. The main definitions regarding Galois correspondences and GLs are presented in [3]. Other results on GL redefined in the field of FCA appear in [10]. Herein, we use the broad presentation given in [13].

\subsection{RET Detection Process}

1. Pre-processing: Fuzzy and alpa-cuts context construction,

2. Generation of closed sets and calculation of associated fuzzy sets,

3. For each detection vector do,

4. associate closed sets with extent (TSs) containing it,

5. For each closed set compute the fuzzy cardinality,

6. Select the closed set with maximum fuzzy cardinality and associate the intent (RET) with the detection vector.

The data table below represents an example of the RET as a fuzzy formal context (from Table 1)

We have:

(D) $\sigma_{1}=\left\{\gamma_{1} / 0.22, \gamma_{2} / 0.18, \gamma_{3} / 0.07, \gamma_{4} / 0.02, \gamma_{5} / 0.28, \gamma_{6} / 0.13, \gamma_{7} / 0.01, \gamma_{8} / 0.001, \gamma_{9} / 0.05\right\}$

$(R) \sigma_{2}=\left\{\gamma_{1} / 0.03, \gamma_{2} / 0.025, \gamma_{3} / 0.08, \gamma_{4} / 0.04, \gamma_{5} / 0.32, \gamma_{6} / 0.08, \gamma_{7} / 0.005, \gamma_{8} / 0.002, \gamma_{9} / 0.02\right\}$

$(I C) \sigma_{3}=\left\{\gamma_{1} / 0.04, \gamma_{2} / 0.017, \gamma_{3} / 0.055, \gamma_{4} / 0.02, \gamma_{5} / 0.20, \gamma_{6} / 0.015, \gamma_{7} / 0.001, \gamma_{8} / 0.002, \gamma_{9} / 0.04\right\}$ 


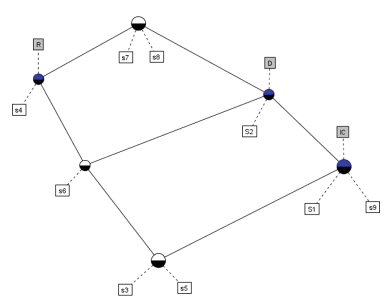

Fig. 1. GL of the context given by Table 1, thresholds level: 0.04

Table 2. RET-fuzzy formal context illustration

\begin{tabular}{l|l}
\hline Fuzzy closed set & Fuzzy set- extent/intent \\
\hline$\left[\gamma_{3}, \gamma_{4}, \gamma_{5}, \gamma_{6} / R\right]$ & $\left\{\gamma_{1} / 0, \gamma_{2} / 0, \gamma_{3} / 0.08, \gamma_{4} / 0.04, \gamma_{5} / 0.32, \gamma_{6} / 0.08, \gamma_{7} / 0, \gamma_{8} / 0, \gamma_{9} / 0\right\}$ \\
\hline$\left[\gamma_{3}, \gamma_{5}, \gamma_{6} / D, R\right]$ & $\left\{\gamma_{1} / 0, \gamma_{2} / 0, \gamma_{3} / 0.07, \gamma_{4} / 0, \gamma_{5} / 0.28, \gamma_{6} / 0.13, \gamma_{7} / 0, \gamma_{8} / 0, \gamma_{9} / 0\right\} \widetilde{\cap}$ \\
& $\left\{\gamma_{1} / 0, \gamma_{2} / 0, \gamma_{3} / 0.08, \gamma_{4} / 0, \gamma_{5} / 0.32, \gamma_{6} / 0.08, \gamma_{7} / 0, \gamma_{8} / 0, \gamma_{9} / 0\right\}$ \\
& $=\left\{\gamma_{1} / 0, \gamma_{2} / 0, \gamma_{3} / 0.07, \gamma_{4} / 0, \gamma_{5} / 0.28, \gamma_{6} / 0.08, \gamma_{7} / 0, \gamma_{8} / 0, \gamma_{9} / 0\right\}$ \\
\hline$\left[\gamma_{3}, \gamma_{5} / R, D, I C\right]$ & $\left\{\gamma_{1} / 0, \gamma_{2} / 0, \gamma_{3} / 0.07, \gamma_{4} / 0, \gamma_{5} / 0.28, \gamma_{6} / 0, \gamma_{7} / 0, \gamma_{8} / 0, \gamma_{9} / 0\right\} \widetilde{n}$ \\
& $\left\{\gamma_{1} / 0, \gamma_{2} / 0, \gamma_{3} / 0.08, \gamma_{4} / 0, \gamma_{5} / 0.32, \gamma_{6} / 0, \gamma_{7} / 0, \gamma_{8} / 0, \gamma_{9} / 0\right\} \widetilde{\cap}$ \\
& $\left\{\gamma_{1} / 0, \gamma_{2} / 0, \gamma_{3} / 0.055, \gamma_{4} / 0, \gamma_{5} / 0.20, \gamma_{6} / 0, \gamma_{7} / 0, \gamma_{8} / 0, \gamma_{9} / 0\right\}$ \\
& $=\left\{\gamma_{1} / 0, \gamma_{2} / 0, \gamma_{3} / 0.055, \gamma_{4} / 0, \gamma_{5} / 0.20, \gamma_{6} / 0, \gamma_{7} / 0, \gamma_{8} / 0, \gamma_{9} / 0\right\}$ \\
\hline$\left[\gamma_{1}, \gamma_{2}, \gamma_{3}, \gamma_{5}, \gamma_{6}, \gamma_{9} / D\right]$ & $\left\{\gamma_{1} / 0.22, \gamma_{2} / 0.18, \gamma_{3} / 0.07, \gamma_{4} / 0, \gamma_{5} / 0.28, \gamma_{6} / 0.13, \gamma_{7} / 0, \gamma_{8} / 0, \gamma_{9} / 0.05\right\}$ \\
\hline$\left[\gamma_{1}, \gamma_{3}, \gamma_{5}, \gamma_{9} / D, I C\right]$ & $\left\{\gamma_{1} / 0.22, \gamma_{2} / 0, \gamma_{3} / 0.07, \gamma_{4} / 0, \gamma_{5} / 0.28, \gamma_{6} / 0, \gamma_{7} / 0, \gamma_{8} / 0, \gamma_{9} / 0.05\right\} \widetilde{n}$ \\
& $\left\{\gamma_{1} / 0.04, \gamma_{2} / 0, \gamma_{3} / 0.055, \gamma_{4} / 0, \gamma_{5} / 0.20, \gamma_{6} / 0, \gamma_{7} / 0, \gamma_{8} / 0, \gamma_{9} / 0.04\right\}$ \\
& $=\left\{\gamma_{1} / 0.04, \gamma_{2} / 0, \gamma_{3} / 0.055, \gamma_{4} / 0, \gamma_{5} / 0.20, \gamma_{6} / 0, \gamma_{7} / 0, \gamma_{8} / 0, \gamma_{9} / 0.04\right\}$ \\
\hline$\left[\gamma_{1}, \gamma_{2}, \gamma_{3}, \gamma_{4}, \gamma_{5}, \gamma_{6}, \gamma_{7}, \gamma_{8}, \gamma_{9} / \emptyset\right]$ & $\left\{\gamma_{1} / 0, \gamma_{2} / 0, \gamma_{3} / 0, \gamma_{4} / 0, \gamma_{5} / 0, \gamma_{6} / 0, \gamma_{7} / 0, \gamma_{8} / 0, \gamma_{9} / 0\right\}$ \\
\hline
\end{tabular}

Table 2 gives the results of browsing within the GL. For each detection vector, we associate the closed (intent/extent) set of the GL, whose intent contains it, or with which the intersection with the intent is not empty. If several closed sets are found, the fuzzy intersection of the intents is performed to determine the extent (Table 3).

Table 3. RET detection process results

\begin{tabular}{|c|c|c|c|}
\hline Detected sequence $(50 \mathrm{~m})$ & Closed set & Fuzzy Sets & RET \\
\hline$\bullet \gamma_{1} \mathbb{P} \gamma_{2} \nabla \gamma_{3}$ & {$\left[\gamma_{1}, \gamma_{2}, \gamma_{3}, \gamma_{5}, \gamma_{9} / D\right]$} & $\left\{\gamma_{1} / 0.22, \gamma_{2} / 0.18, \gamma_{3} / 0.07\right\}$ & $\mathrm{D}$ \\
\hline $\mathbb{P} \gamma_{2} \nabla \gamma_{3} @ \gamma_{4}$ & $\begin{array}{l}{\left[\gamma_{3}, \gamma_{4}, \gamma_{5}, \gamma_{6} / R\right]} \\
{\left[\gamma_{1}, \gamma_{2}, \gamma_{3}, \gamma_{5}, \gamma_{9} / D\right]}\end{array}$ & $\begin{array}{l}\left\{\gamma_{3} / 0.08, \gamma_{4} / 0.04\right\} / R[0.12] \\
\left\{\gamma_{2} / 0.18, \gamma_{3} / 0.07\right\} / D[0.25]\end{array}$ & $\mathrm{D}$ \\
\hline (ख) $\gamma_{4} \quad T \gamma_{5}$ & {$\left[\gamma_{3}, \gamma_{4}, \gamma_{5}, \gamma_{6} / R\right]$} & $\left\{\gamma_{4} / 0.04, \gamma_{5} / 0.32\right\} / R$ & $\mathrm{R}$ \\
\hline$\nabla \gamma_{3}+\gamma_{9}$ & $\begin{array}{l}{\left[\gamma_{1}, \gamma_{2}, \gamma_{3}, \gamma_{5}, \gamma_{9} / D\right]} \\
{\left[\gamma_{1}, \gamma_{3}, \gamma_{5}, \gamma_{9} / D, I C\right]}\end{array}$ & $\begin{array}{l}\left\{\gamma_{3} / 0.07, \gamma_{9} / 0.05\right\} / D[0.12] \\
\left\{\gamma_{3} / 0.055, \gamma_{9} / 0.04\right\} / D, I C[0.095]\end{array}$ & $\mathrm{D}$ \\
\hline$\gamma_{1}+\gamma_{5}$ & $\begin{array}{l}{\left[\gamma_{1}, \gamma_{2}, \gamma_{3}, \gamma_{5}, \gamma_{9} / D\right]} \\
{\left[\gamma_{1}, \gamma_{3}, \gamma_{5}, \gamma_{9} / D, I C\right]}\end{array}$ & $\begin{array}{l}\left\{\gamma_{3} / 0.07, \gamma_{9} / 0.05\right\} / D[0.12] \\
\left\{\gamma_{3} / 0.055, \gamma_{9} / 0.04\right\} / D, I C[0.095]\end{array}$ & D \\
\hline
\end{tabular}

\subsection{FCA Process and Architecture}

In this section, we describe the use of the closed GL associated with the relationship expressing the dependencies between the TSs and the CRs, on the one 
hand, and RET, on the other hand. The exploration process starts with the collection of various data from the road environments observed within the urban network. This relatively large volume of data is then exploited to form probabilities and matrix structures defining the dependencies, or couplings between the elements being in relation. This step requires the choice of objects and attributes in the (TS, CR) x RET repository. These structures materialize as fuzzy contexts, which will be subsequently thresholded (i.e., binarized according to some thresholds) to create binary Galois correspondences. Then these contexts will be used to generate GLs and thus lead to the exploration according to the holistic exploration practice as presented in Fig. 1. It shows an illustrative example of the process and the corresponding software architecture.

\section{Conclusion}

In this paper, an FCA approach was applied to the problem of urban RET detection. Our purpose was to characterize the road environment - swishing past around an ego-car - based on TS and CR occurrences detected by a TSR system. The results obtained by browsing GL closed sets provide insight to the detection intricacies. The results of the pilot-study was rather promising, but large-scale tests are required to thoroughly validate the approach.

\section{References}

1. Andor, C., Joo, A., Mero, L.: Galois-lattices: a possible representation of knowledge structures. Eval. Educ. 9(2), 207-215 (1985)

2. Antoni, L., Krajči, S., Krídlo, O.: Representation of fuzzy subsets by Galois connections. Fuzzy Sets Syst. 326, 52-68 (2017)

3. Barbut, M., Monjardet, B.: Ordre et Classification. Algèbre et Combinatoire, Tomes I et II, Paris, Hachette (1970)

4. Birkhoff, G.: Lattice theory, American Mathematical Society Colloquium Publications, vol. XXV, 1st ed., 1940, 3rd ed. (1967)

5. Fazekas, Z., Balázs, G., Gerencsér, L., Gáspár, P.: Inferring the actual urban road environment from traffic sign data using a minimum description length approach. Transp. Res. Proc. 27, 516-523 (2017)

6. Fazekas, Z., Balázs,G., Gerencsér, L., Gáspár, P.: Detecting change in the urban road environment along a route based on traffic sign and crossroad data. In: Intelligent Transport Systems - From Research and Development to the Market Uptake, LNICST, Social Informatics amp; Telecommunications Engineering, vol. 222, pp. 252-262. Springer, (2018)

7. Fazekas, Z., Balázs, G., Gáspár, P.: ANN-based classification of urban road environments from traffic sign and crossroad data. Acta Polytech. Hung. 15, 83-100 (2018)

8. Fazekas, Z., Balázs, G., Gáspár, P.: Building upon modularity in artificial neural networks. ERCIM News 116, 30-31 (2019)

9. Fazekas, Z., Balázs, G., Gáspár, P.: A heuristic approach to road environmenttype detection from traffic sign data. In: Proceedings of the 4th IEEE Conference on Control and Fault Tolerant Systems, Casablanca, Morocco, pp. 288-293. IEEE (2019) 
10. Ganter, B., Wille, R.: Formal Concept Analysis: Mathematical Foundations. Springer, New York (1997). ISBN 3540627715

11. Le Ber, F., Napoli, A.: A Galois lattice for qualitative spatial reasoning and representation. In: Proceedings of the ECAI Workshop on Advances in Formal Concept Analysis for Knowledge Discovery in Databases, Lyon, France, 12 p. (2002)

12. Vishnukumar, H.J., Butting, B., Müller, C., Sax, E.: Machine learning and deep neural network - artificial intelligence core for lab and real-world test and validation for ADAS and autonomous vehicles: AI for efficient and quality test and validation. In: Intelligent Systems Conference, London, UK, pp. 714-721. IEEE (2017)

13. Wille, R.: Concept lattices and conceptual knowledge systems. Comput. Math. Appl. 23(6-9), 493-515 (1992)

14. Wolff, K.E.: Concepts in fuzzy scaling theory: order and granularity. Fuzzy Sets Syst. 132(1), 63-75 (2002)

15. Zimmermann, H.J.: Fuzzy Set Theory and Its Applications, 4th edn. Springer, Heidelberg (2001) 